\title{
FUNCTIONAL CONNECTIVITY EIGENNETWORKS REVEAL DIFFERENT BRAIN DYNAMICS IN MULTIPLE SCLEROSIS PATIENTS
}

\author{
Nora Leonardi ${ }^{1,2}$, Jonas Richiardi ${ }^{1,2,3}$, Dimitri Van De Ville ${ }^{1,2}$ \\ ${ }^{1}$ Medical Image Processing Lab, Ecole Polytechnique Fédérale de Lausanne, Lausanne, CH \\ ${ }^{2}$ Department of Radiology and Medical Informatics, University of Geneva, Geneva, CH \\ ${ }^{3}$ Functional Imaging in Neuropsychiatric Disorders Lab, Stanford University, Palo Alto, USA
}

\begin{abstract}
Resting state functional connectivity is defined as correlations in brain activity measured by functional magnetic resonance imaging without any stimulation paradigm. Such connectivity is dynamic, even over the course of minutes, and the development of tools for its analysis is an important challenge in neuroscience. We propose a novel data-driven technique to extract connectivity patterns from dynamic whole-brain networks of multiple subjects. Our technique is based on singular value decomposition and decomposes a collection of networks into linearly independent "eigennetworks" and associated time courses. To deal with the temporal redundancy of networks, we propose a novel subsampling method based on the standard deviation of the connectivity strength. We apply the proposed technique to dynamic resting-state networks of healthy subjects and multiple sclerosis patients, and show its potential to detect aberrant connectivity patterns in patients.
\end{abstract}

Index Terms - fMRI, dynamic functional connectivity, complex networks, matrix decomposition, multiple sclerosis

\section{INTRODUCTION}

Understanding how human brain networks evolve over time, even across time spans as short as a couple of minutes, is a crucial challenge in neuroscience. Functional brain networks are typically estimated from functional magnetic resonance imaging (fMRI) data, which is a proxy for brain activity. Functional connectivity (FC) is defined as temporal correlations between the activity of distinct brain areas, and resting-state (RS) FC is estimated from data in the absence of a task. Interestingly, the activity of areas with similar functional properties, such as the primary motor cortex, is also coherent during RS [1]. Similar results have since been reported for other functional networks, leading to novel insights about the functional organisation of the brain. Most studies assume FC to be stationary across the duration of a RS scan. However, recent studies have shown that RSFC is highly dynamic

This work was supported by the Swiss National Science Foundation (grant PP00P2-123438), the Société Académique de Genève, the FOREMANE foundation, the Center for Biomedical Imaging (CIBM).
$[2,3,4]$ and that these fluctuations persist in the absence of conscious, cognitive processing in anesthetized monkeys [5]. These results suggest that the dynamic nature of RSFC reflects an inherent property of the brain's functional organization and that common stationary analyses are an oversimplification. Most studies analyzing dynamic FC focus on a single brain region or functional network and how its connectivity changes over time. Discovering FC patterns in dynamic whole-brain networks is a challenging task. Since the nature of RS is unconstrained, any patterns need to be identified in a data-driven manner. Allen et al. [6] recently extracted connectivity states from dynamic whole-brain RSFC networks in a data-driven manner using $k$-means clustering.

In this work, we propose a novel approach to extract connectivity patterns from multi-subject, dynamic whole-brain FC networks. Our technique is based on the singular value decomposition (SVD) and decomposes a set of networks into "eigennetworks", or building blocks of functional connectivity, and associated time courses. To deal with the redundancy of networks that are close in time, we subsample the set of networks by retaining only networks at "peak times", which we define as those having a large standard deviation of FC strength. This selection procedure is inspired by electroencephalogram (EEG) topographical analysis, where global field power is computed as standard deviation of voltages across electrodes [7].

We apply the proposed technique to dynamic RSFC networks from healthy subjects and minimally disabled relapseremitting multiple sclerosis (MS) patients. MS is an autoimmune disease of the central nervous system and disturbs both structural and functional connectivity [8]. Altered stationary FC has been linked with disease progression and cognitive impairment in MS patients and the analysis of RSFC promises to yield novel insights into the functional impact of MS $[9,10]$.

The paper is organized as follows. In Sect. 2, we describe the complete data processing and analysis pipeline. In Sect. 3, we demonstrate the potential of the proposed approach to identify differences in RSFC dynamics between controls and patients. 


\section{ESTIMATION OF EIGENNETWORKS}

\subsection{Data processing and dynamic FC estimation}

Functional imaging data was realigned and motion-corrected using SPM8, linearly detrended, low-pass filtered and nuisance variables regressed out (6 head realignment parameters, averaged cerebrospinal fluid and white matter signal). The anatomical image was parcellated into $N=90$ regions corresponding to the Automated Anatomic Labeling atlas [11]. Due to signal dropout in ventral regions, the globus pallidus was excluded from the analysis, resulting in $N=88$ regions being kept. Regional time series were estimated by averaging the functional data in each anatomical region.

To estimate dynamic connectivity we used a slidingwindow technique [2]. We computed pair-wise windowed interregional correlations $\rho_{x y}(t)=\operatorname{corr}(x[t, t+\Delta t], y[t, t+$ $\Delta t]$ ), where $t=1,2, \ldots, 400$ and $\Delta t=40$ is the window size, which includes typical RSFC fluctuations $[4,5,6]$. Each $N \times N$ correlation matrix A was approximately Gaussianized by using the Fisher R-to-Z transform and normalized by its $\ell_{1}$-norm.

\subsection{Estimation of connectivity patterns}

We describe the set of dynamic networks by vectorizing and concatenating the correlation matrices across windows and subjects to form a $\frac{N^{2}-N}{2} \times T S$ matrix $\mathcal{A}=$ $\left[\operatorname{triu}\left(\mathbf{A}_{1}\right)\left|\operatorname{triu}\left(\mathbf{A}_{2}\right)\right| \ldots \mid \operatorname{triu}\left(\mathbf{A}_{T S}\right)\right]$, where we keep only the upper triangular part because of symmetry, $T$ is the number of windows and $S$ the number of subjects (Fig. 1). Since there is no temporal correspondence across subjects for RS scans, we concatenated the correlation vectors $\mathbf{a}=\operatorname{triu}(\mathbf{A})$ across subjects instead of forming a third-order array.

Because of the sliding-window technique adjacent correlation matrices are similar. To deal with this redundancy, we propose to subsample the $T S$ networks using an approach inspired by EEG topographical analysis [7]: We retained correlation vectors a corresponding to peaks of "global connectivity power" (GCP), where GCP was defined as the standard deviation of each a, and peaks as local maxima of GCP. GCP is high for correlation vectors with pronounced variability and low for ones with similar strength (Fig. 1).

We then balanced the number of vectors a in the healthy and patient groups by randomly subsampling the patient group. We decomposed the reduced matrix containing only the correlation vectors marked as peaks after subsampling in the patient group using SVD, i.e., $\mathcal{A}_{\text {reduced }}=\mathbf{U S V}^{T}$, where $\mathbf{U}$ contains spatial maps of connectivity patterns, which we call eigennetworks and that can be interpreted as building blocks of dynamic RSFC, and $\mathbf{V}$ the associated time courses. We then projected $\mathcal{A}$ onto the eigennetworks, i.e., $\mathbf{T}=\mathbf{U}^{T} \mathcal{A}$, to obtain time courses of length $T$ for each subject and eigennetwork. We tested for significant differences in the time course means associated with the first 10 eigennetworks of

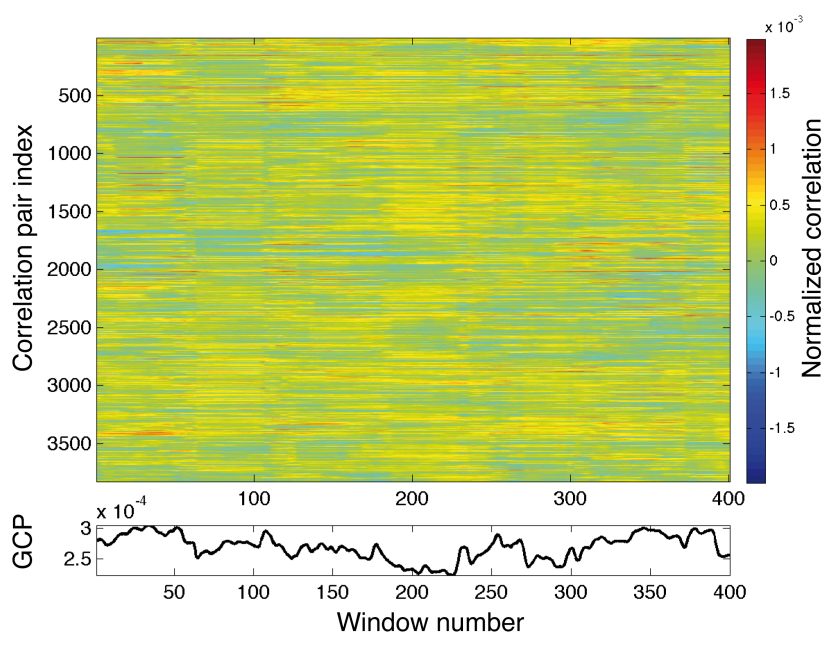

Fig. 1: Example dynamic functional connectivity estimation for a healthy subject: vectorized and temporally concatenated correlation matrices; and GCP of each correlation vector.

the two groups using Hotelling's $\mathrm{T}^{2}$ multivariate test and posthoc two-sample $t$ tests. We compared only the mean since individual windows can not be assumed to be consistent across subjects.

From the loadings $\mathrm{w}$ of Hotelling's $\mathrm{T}^{2}$ test, we constructed a difference network, i.e., the weighted sum $\mathbf{U}_{[1,10]} \mathbf{w}$ across the first 10 eigennetworks, where the weights were such that the group difference was maximised: $\max _{\mathbf{w}} \mathbf{w}^{T} \boldsymbol{\mu}^{H C}-\mathbf{w}^{T} \boldsymbol{\mu}^{M S}$, s.t. $\|\mathbf{w}\|=1$, with $\boldsymbol{\mu}$ the vector of time course means.

\subsection{Relationship with motion}

To investigate relationships between motion and nonstationarity in whole-brain connectivity, we converted each subject's head realignment parameters into two measures of displacement in 3-D space between adjacent scans: $D_{1}=$ $\sqrt{\Delta x^{2}+\Delta y^{2}+\Delta z^{2}}$, and $D_{2}=|\Delta x|+|\Delta y|+|\Delta z|+$ $|\Delta \alpha|+|\Delta \beta|+|\Delta \gamma|$, where rotational displacements $\alpha, \beta, \gamma$ were converted to millimetres by calculating the displacement on the surface of a sphere of radius $50 \mathrm{~mm}$ [12]. We converted $D_{1}$ and $D_{2}$ to a measure for each window by estimating the intensity (average) and fluctuation of motion (standard deviation) in each window.

We then computed Pearson's correlation coefficient between the motion statistics time series and the GCP peak time series as well as the $\mathbf{V}$ time series for each subject. Statistically significant correlations with motion were determined by estimating a maximal statistic under the null hypothesis: i.e., observed correlations in the $95^{t h}$ percentile of the null distribution of phase randomised time series are significant at the $5 \%$ level ( $n=1^{\prime} 000$ randomisations, corrected $\mathrm{p}$ value) [13]. Phase-randomised time series preserve mean, variance and autocorrelation properties [4, 14]. 


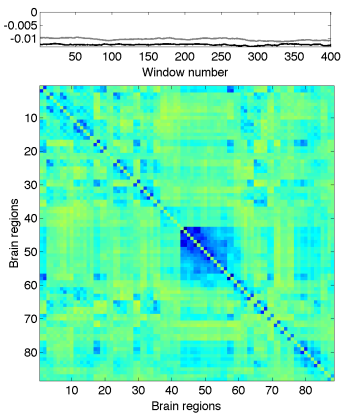

(a) Eigennetwork 1

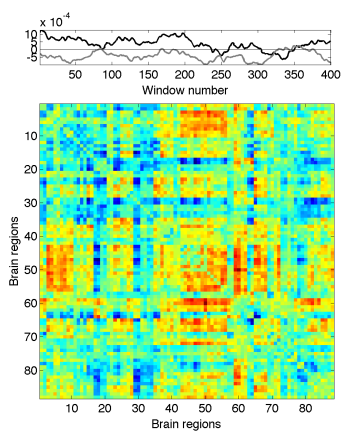

(f) Eigennetwork 6

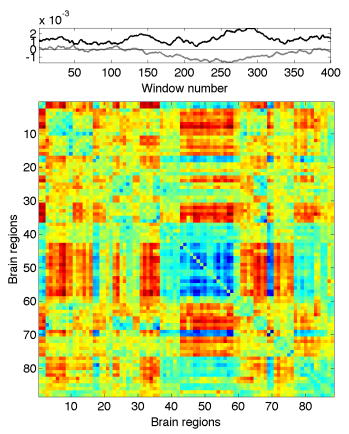

(b) Eigennetwork 2

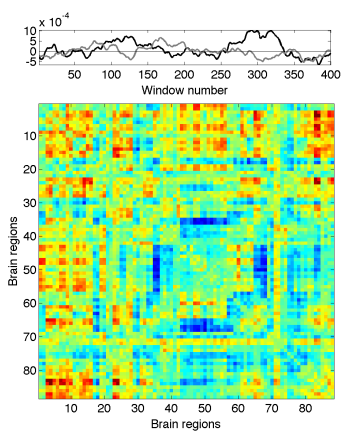

(g) Eigennetwork 7

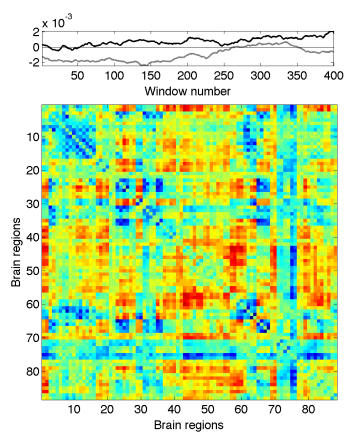

(c) Eigennetwork 3

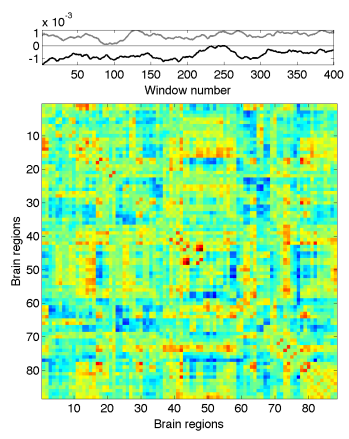

(h) Eigennetwork 8

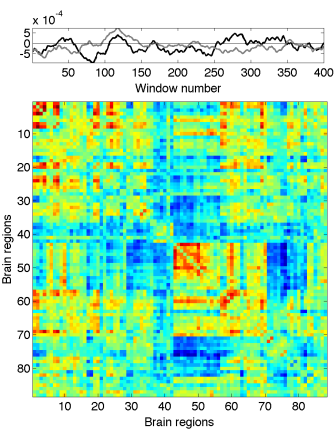

(d) Eigennetwork 4

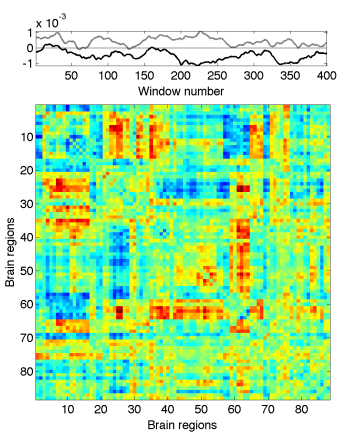

(i) Eigennetwork 9

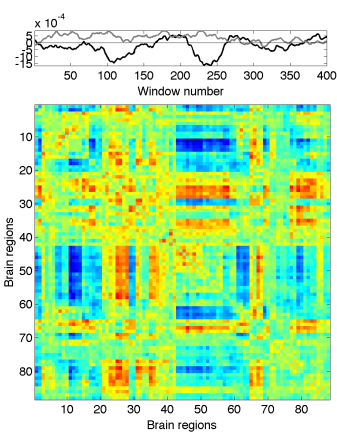

(e) Eigennetwork 5

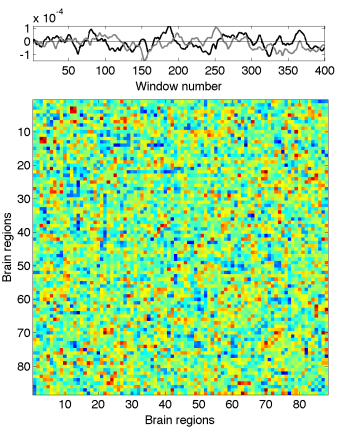

(j) Eigennetwork 500

Fig. 2: Eigennetworks $1-9$ and 500 and the average time course of healthy subjects (black line) and MS patients (gray line). The columns of $\mathbf{U}$ were reshaped as upper triangular matrices and symmetrized. Colorbars are symmetric around zero, red thus corresponds to positive and blue to negative signs. Brain regions are arranged with homologous regions adjacent to each other and are ordered by lobe (central $1,2,57,58$, frontal $3-28$, limbic $29-42$, occipital $43-56$, parietal $59-68$, subcortical $71-76$, temporal $77-88$ ).

\section{EXPERIMENTAL RESULTS}

\subsection{Participants and data acquisition}

The dataset included 13 healthy subjects and 22 minimally disabled relapsing-remitting MS patients (mean \pm SD Expanded Disability Status Scale $1.9 \pm 0.4$ ) [10]. The resting state functional scan was acquired on a Siemens 3T TrioTIM $\left(\mathrm{TR} / \mathrm{TE} / \mathrm{FA}=1.1 \mathrm{~s} / 27 \mathrm{~ms} / 90^{\circ}\right.$, matrix $=64 \times 64$, voxel size $=$ $3.75 \times 3.75 \times 5.63 \mathrm{~mm}^{3}, 21$ contiguous transverse slices, 450 volumes). Subjects were instructed to lie still with their eyes closed and to let their mind wander. The anatomical scan was acquired using a 3-D T1-weighted MPRAGE sequence $\left(160\right.$ slices, $\mathrm{TR} / \mathrm{TE} / \mathrm{FA}=2.4 \mathrm{~s} / 2.98 \mathrm{~ms} / 9^{\circ}$, matrix $=256 \times 240$, voxel size $=1 \times 1 \times 1.2 \mathrm{~mm}^{3}$ ).

\subsection{Eigennetworks}

The 4 motion measures were not significantly different between healthy subjects and MS patients $(p>0.05)$ and not significantly correlated with GCP $(p>0.05)$.

Fig. 2 shows some of the connectivity patterns captured by the SVD, which represent connections with coherent (same sign) or incoherent fluctuations (different signs).
The first eigennetworks, which explain most of the variance across the dynamic FC matrices, identify large-scale structures. The average network contains well-known functional clusters (Fig. 2a): a strongly connected visual cortex (nodes $43-56)$, auditory processing areas $(79-86)$, and motor areas $(1,2,19,20,57,58)$. Coherently fluctuating connections are for example connections between the visual cortex $(43-56)$ and motor areas $(1,2,17,18,57,58,69,70$, blue in eigennetwork 2) or between regions belonging to the default mode network $(23-26,31,35,36,65,67,68$, red in eigennetwork $5)$. Eigennetworks of very high components correspond to noise (Fig. 2j). The time courses of the eigennetworks (i.e., the corresponding columns in $\mathbf{V}$ ) were not significantly correlated with the 4 motion measures $(p>0.05)$.

The average projected time course $\mathbf{T}$ of each group is shown atop the eigennetworks in Fig. 2 and represents the contribution of this eigennetwork or building block to the correlation matrix at any given window. Even though RS behavior cannot be assumed temporally consistent between subjects, visual inspection of the mean time courses reveals some differences already. Next, we tested for significant differences in the mean of the 10 first time courses between the two groups using Hotelling's $\mathrm{T}^{2}$ test. (The subset of 10 eigen- 


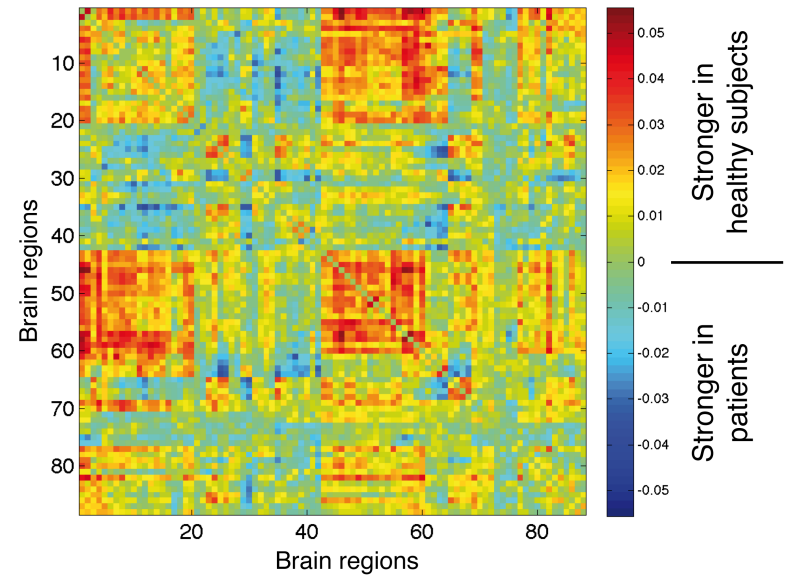

Fig. 3: Discriminative network: weighted sum of eigennetworks $1-10$, where the weights were according to their importance in separating the two groups in Hotelling's $\mathrm{T}^{2}$ test.

networks retained $49 \%$ of the total variance.) Hotelling's $\mathrm{T}^{2}$ test detected significant group $(p=0.006)$ and interaction effects $(p=0.004)$. Post-hoc t tests identified differences in eigennetworks 1 and 8 ( $p<0.001, p=0.01$, respectively). We observe that (1) the overall connectivity strength is reduced in patients $\left(\left|\mu_{1}^{M S}\right|<\left|\mu_{1}^{H C}\right|\right.$, Fig. 2a), in accordance with previous studies $[9,10]$, and (2) eigennetwork 8 captures coherent dynamic connectivity that is either increased in patients (red areas in Fig. 2h since $\mu_{8}^{M S}>0$ ) or healthy subjects (blue areas since $\mu_{8}^{H C}<0$ ).

The difference network, which highlights differently contributing eigennetworks, shows that most regions are more strongly connected in healthy subjects (Fig. 3). To identify the brain regions with the largest connectivity differences, we calculated the degree of each brain region by summing over all its weighted connections. Regions with increased connectivity in healthy subjects included the pre- and postcentral gyri $(1,2,57,58)$, the cuneus $(45,46)$, lingual gyrus $(47,48)$, superior parietal gyrus $(59,60)$, right superior temporal pole (82), and left paracentral lobule (69). Regions with increased connectivity in patients included the right insula (30), right amygdala (42), left posterior cingulate gyrus (35), left thalamus (75), and left parahippocampus (39).

\section{CONCLUSION}

We have presented a novel approach to identify whole-brain FC patterns, so-called "eigennetworks", from dynamic networks in a data-driven manner, and shown that they represent meaningful functional clusters. The comparison of FC dynamics between healthy subjects and MS patients revealed differences in the contribution of these eigennetworks across the duration of the scan. While the stationary FC profile of some of these regions has previously been reported to be discriminative between the two groups [10], our analysis of dy- namic FC complements this study by identifying further regions with aberrant FC. The proposed data-driven technique for dynamic brain networks holds promise for the study of how brain dynamics are altered in disease.

\section{ACKNOWLEDGEMENTS}

The authors thank P. Schluep, S. Simioni, J.-M. Annoni, M. Gschwind and P. Vuilleumier for sharing the data.

\section{REFERENCES}

[1] B. Biswal, F. Z. Yetkin, V. M. Haughton, and J. S. Hyde, "Functional connectivity in the motor cortex of resting human brain using echo-planar mri.," Magn Reson Med, vol. 34, no. 4, pp. 537-541, Oct 1995.

[2] C. Chang and G. Glover, "Time-frequency dynamics of resting-state brain connectivity measured with fMRI," Neurolmage, vol. 50, no. 1, pp. 81-98, Mar. 2010.

[3] V. Kiviniemi, T. Vire, J. Remes, A. A. Elseoud, T. Starck, O. Tervonen, and J. Nikkinen, "A sliding time-window ICA reveals spatial variability of the default mode network in time," Brain Connect, vol. 1, no. 4, pp. 339-347, Oct 2011.

[4] D. A. Handwerker, V. Roopchansingh, J. Gonzalez-Castillo, and P. A. Bandettini, "Periodic changes in fMRI connectivity," Neuroimage, vol. 63, no. 3, pp. 1712-1719, Jul 2012.

[5] R. M. Hutchison, T. Womelsdorf, J. S. Gati, S. Everling, and R. S. Menon, "Resting-state networks show dynamic functional connectivity in awake humans and anesthetized macaques," Hum Brain Mapp, Mar 2012.

[6] E. A. Allen, E. Damaraju, S. M. Plis, E. B. Erhardt, T. Eichele, and V. D. Calhoun, "Tracking whole-brain connectivity dynamics in the resting state," Cerebral Cortex, 2012.

[7] R. D. Pascual-Marqui, C. M. Michel, and D. Lehmann, "Segmentation of brain electrical activity into microstates: model estimation and validation.," IEEE Trans Biomed Eng, vol. 42, no. 7, pp. 658-665, Jul 1995.

[8] M. Filippi, F. Agosta, E. G. Spinelli, and M. A. Rocca, "Imaging resting state brain function in multiple sclerosis.," J Neurol, Oct 2012.

[9] M. A. Rocca, P. Valsasina, M. Absinta, G. Riccitelli, M. E. Rodegher, P. Misci, P. Rossi, A. Falini, G. Comi, and M. Filippi, "Default-mode network dysfunction and cognitive impairment in progressive MS," Neurology, vol. 74, no. 16, pp. 1252-1259, Apr 2010.

[10] J. Richiardi, M. Gschwind, S. Simioni, J.-M. Annoni, B. Greco, P. Hagmann, M. Schluep, P. Vuilleumier, and D. Van De Ville, "Classifying minimally disabled multiple sclerosis patients from resting state functional connectivity," NeuroImage, vol. 62, no. 3, pp. 2021-2033, Sep 2012.

[11] N. Tzourio-Mazoyer, B. Landeau, D. Papathanassiou, F. Crivello, O. Etard, N. Delcroix, B. Mazoyer, and M. Joliot, "Automated anatomical labeling of activations in SPM using a macroscopic anatomical parcellation of the MNI MRI singlesubject brain," NeuroImage, vol. 15, no. 1, pp. 273-289, Jan. 2002.

[12] J. D. Power, K. A. Barnes, A. Z. Snyder, B. L. Schlaggar, and S. E. Petersen, "Spurious but systematic correlations in functional connectivity MRI networks arise from subject motion," Neuroimage, vol. 59, no. 3, pp. 2142-2154, Feb 2012.

[13] T. E. Nichols and A. P. Holmes, "Nonparametric permutation tests for functional neuroimaging: a primer with examples.," Hum Brain Mapp, vol. 15, no. 1, pp. 1-25, Jan 2002.

[14] J. Theiler, S. Eubank, A. Longtin, B. Galdrikian, and J. D. Farmer, "Testing for nonlinearity in time series: the method of surrogate data," Physica D: Nonlinear Phenomena, vol. 58, no. 14 , pp. $77-94,1992$. 